Article

\title{
Electrochemical Aptasensor for Detection of Dopamine
}

\author{
Hisham Abu-Ali ${ }^{1,3}$, Cansu Ozkaya ${ }^{1,2}$, Frank Davis ${ }^{1}{ }^{\mathbb{D}}$, Nik Walch ${ }^{1}$ and Alexei Nabok ${ }^{1, *}$ \\ 1 Department of Engineering and Mathematics, Materials and Engineering Research Institute, Sheffield \\ Hallam University, City Campus, Howard Street, Sheffield S1 1WB, UK; hishamfaiadh@yahoo.com (H.A.-A.); \\ cansuzky@gmail.com (C.O.); frankdavis1966@googlemail.com (F.D.); njwalch@gmail.com (N.W.) \\ 2 Department of Physics, Balikesir University, 10145 Cagis, Balıkesir, Turkey \\ 3 College of Science, Biology Department, University of Basrah, Basrah IQ-61002, Iraq \\ * Correspondence: a.nabok@shu.ac.uk
}

Received: 27 February 2020; Accepted: 11 April 2020; Published: 15 April 2020

check for updates

\begin{abstract}
This work presents a proof of concept of a novel, simple, and sensitive method of detection of dopamine, a neurotransmitter within the human brain. We propose a simple electrochemical method for the detection of dopamine using a dopamine-specific aptamer labeled with an electrochemically active ferrocene tag. Aptamers immobilized on the surface of gold screen-printed gold electrodes via thiol groups can change their secondary structure by wrapping around the target molecule. As a result, the ferrocene labels move closer to the electrode surface and subsequently increase the electron transfer. The cyclic voltammograms and impedance spectra recorded on electrodes in buffer solutions containing different concentration of dopamine showed, respectively, the increase in both the anodic and cathodic currents and decrease in the double layer resistance upon increasing the concentration of dopamine from 0.1 to $10 \mathrm{nM} \mathrm{L}^{-1}$. The high affinity of aptamer-dopamine binding (KD $\approx 5 \mathrm{nM}$ ) was found by the analysis of the binding kinetics. The occurrence of aptamer-dopamine binding was directly confirmed with spectroscopic ellipsometry measurements.
\end{abstract}

Keywords: dopamine; electrochemical biosensor; aptamer; cyclic voltammetry; electrochemical impedance spectroscopy; spectroscopic ellipsometry

\section{Introduction}

Neurotransmitters are chemical messengers that enable neurons to communicate with each other or to stimulate responses from glandular or muscle cells [1,2]. One important member of this family is dopamine (3,4-dihydroxyphenethylamine), a member of the phenethylamine and catecholamine families of compounds. It plays several important roles in the brain and the central nervous system as well as in the cardiovascular, renal, and hormonal systems [1,2]. Control of dopamine levels is extremely important; for example, the lack of dopamine is an indicator of Parkinson's disease. Other neurological disorders such as schizophrenia and Alzheimer's also demonstrate abnormal dopamine levels [1,2]. It is obvious therefore that monitoring dopamine levels is necessary in the diagnostics and treatment of these conditions.

The detection of dopamine in blood or urine samples is typically done in specialized laboratories using methods such as ELISA (enzyme-linked immunosorbent assay) as well as other spectrometric, fluorescence, colorimetric, and electrochemical methods. However, these tests often require complex laboratory equipment and are expensive and time consuming. A simple point-of-care test would greatly reduce the time and cost of dopamine determination.

Electrochemical methods have been proposed due to their relatively high speed of response, specificity and the use of relatively simple and inexpensive equipment. A large amount of work has been 
done on the electrochemical detection of dopamine and other neurotransmitters [2,3]. Dopamine can be very easily detected in an aqueous solution by electrochemical methods such as cyclic voltammetry since dopamine undergoes electrochemical oxidation. However, a major issue is the selectivity since complex matrices such as blood contain a range of other oxidizable compounds such as urate or ascorbate which would also generate signals, leading to inaccurate readings. Selectivity towards dopamine is therefore required.

A wide range of electrode materials have been proposed to increase the selectivity of dopamine detection [2,3]. A few recent examples include the use of materials such as Nafion combined with graphite [4] or multi-walled carbon nanotubes [5] to improve the selectivity towards dopamine. Other researchers have utilized graphene-modified screen-printed electrodes [6] or graphene aerogels [7] as substrates for the selective determination of dopamine. Composites of carbon nanotubes and graphene oxide could be used to determine dopamine, nitrate, ascorbate, and urate [8]. Metal nanoparticles have also been utilized, for example palladium nanoparticles combined with graphene [9], which were used as a base of electrochemical determination of dopamine whereas a gold nanoparticle/DNA/polymer composite could be used for the simultaneous determination of dopamine, uric acid, guanine, and adenine [10]. Composites of gold nanoparticles with conducting polymers have also been used for the selective determination of dopamine [11]. Boron-doped carbon nanotubes could be used to determine levels of dopamine and ephedrine in the presence of urate [12]. Composites of graphene oxide with $\mathrm{Bi}_{2} \mathrm{~S}_{3}$ nanorods were used for the electrochemical determination of dopamine [13], and arrays of cylindrical gold nanoelectrodes could be used for both the detection of dopamine and for the immobilization and growth of human neural cells [14]. Many other systems based on organic and inorganic materials have been reported for the sensing of dopamine [2,3].

Synthetic bio-receptors such as aptamers appeared to be highly attractive for selective detection of dopamine [15-17]. Aptamers are artificial, relatively short single strand DNA or RNA oligomers having a particular sequence of nucleotides designed to accommodate a target molecule, e.g., dopamine. On binding their target, the aptamers adopt a conformational change from a relatively simple chain structure into a complex 3D shape that wraps around the desired target. A combination of high selectivity of biological systems with the stability and possibly for large scale synthesis of artificial systems make aptamers especially attractive for a wide range of applications; several reviews on the properties and applications of aptamers have been published recently [15-17].

Several research groups have studied the use of aptamers as the recognition elements in dopamine sensors. A 58-nucleotide aptamer could be combined with gold nanoparticles to give a colorimetric sensor for dopamine where the process of aptamer-dopamine binding facilitates aggregation of gold nanoparticles and subsequent color change [18]; a linear detection range of $5.4 \times 10^{-7} \mathrm{M}$ to $5.4 \times 10^{-6} \mathrm{M}$ and a detection limit of $3.6 \times 10^{-7} \mathrm{M}$ were reported. In other work, aptamers for dopamine and gold nanoparticles were combined; the resultant composite underwent fluorescence resonance energy transfer with rhodamine B, quenching its fluorescence. Addition of dopamine prevented this, allowing the detection of dopamine between $2.6 \times 10^{-8} \mathrm{M}$ to $2.9 \times 10^{-6} \mathrm{M}$ with a detection limit of $2 \times 10^{-9} \mathrm{M}[19]$. Surfaces modified with small neurotransmitters such as tryptophan or dopamine have been shown to selectively bind aptamers from solution [20].

Electrochemical methods have also proved suitable. An aptamer for dopamine could be electrostatically adsorbed onto an aminothiol modified gold surface. Binding of dopamine at such aptamer-modified gold electrodes allowed the detection of dopamine by direct oxidation in the physiologically relevant concentration range of $1 \times 10^{-7} \mathrm{M}$ to $5 \times 10^{-6} \mathrm{M}$ [21]. In other work, gold nanoparticles could be chemically modified with a single-stranded DNA that was complementary to a dopamine aptamer [22]. Addition of the aptamer led to formation of the duplex on the nanoparticle surface. In addition to dopamine, the duplex was disrupted with the aptamer binding to dopamine, the gold nanoparticle is released and adsorbs onto a carbon nanotube modified electrode and detected by differential pulse voltammetry, allowing determination of dopamine at levels as low as $1 \times 10^{-8} \mathrm{M}$. A glassy carbon electrode modified with gold and platinum nanoparticles could also be used to 
immobilize a single stranded DNA complementary to a dopamine aptamer, addition of the aptamer caused the formation of duplex that could then act as a host for intercalated methylene blue, which is detectable by differential pulse voltammetry [23]. Addition of dopamine caused disruption of the duplex, release of methylene blue and subsequent decrease in the electrochemical signal. Dopamine could be determined between $1 \times 10^{-9} \mathrm{M}$ to $3 \times 10^{-8} \mathrm{M}$ with the detection limit of $2.2 \times 10^{-10} \mathrm{M}$.

In the above cases, the use of aptamers combined with either optical or electrochemical indicators and sometimes oligonucleotide counter-strands was required. In this work, we report on a simple electrochemical detection of dopamine using a specific aptamer labelled with redox group (ferrocene). This approach was utilized earlier by Marty for detection of mycotoxins, particularly ochratoxin [24], and it was recently successfully adapted for detection of heavy metal ions $\left(\mathrm{Hg}^{2+}\right.$ and $\left.\mathrm{Pb}^{2+}\right)$ in water [25]. This work is therefore focused on expanding the principle of electrochemical sensing using redox-labelled aptamers for detection of dopamine.

\section{Materials and Methods}

\subsection{Aptamers and their Immobilization.}

A DNA-based aptamer against dopamine was obtained from Sangon Biotechnology Co. Ltd. (Shanghai, China) has the following sequence of nucleotides [18]: C5'-GTC TCT GTG TGC GCC AGA GAA CAC TGG GGC AGA TAT GGG CCA GCA CAG AAT GAG GCC C-C3' and was functionalized with thiol groups and ferrocene labels at $\mathrm{C}^{\prime}{ }^{\prime}$ and $\mathrm{C}^{\prime}$ termini respectively. The aptamers were immobilized on the surface of gold screen-printed electrodes following the protocol described previously [25]. Stock solution of the required aptamer was diluted to $1 \mu \mathrm{M}$ in phosphate binding buffer (PBB) supplemented with $1 \mathrm{mM}$ of 1,4-dithiothretiol (DTT) and $3 \mathrm{mM}$ of $\mathrm{MgCl}_{2}$. The addition of DTT led to splitting of the S-S bridge joining two aptamers subsequently releasing thiol (SH) end-groups for binding to the gold surface, while the $\mathrm{Mg}^{2+}$ ions stabilize the secondary structure of the aptamer. Before immobilization, the aptamer solution was activated by rapid ( $1 \mathrm{~min})$ heating up to $95^{\circ} \mathrm{C}$ followed by $1 \mathrm{~min}$ cooling at $4{ }^{\circ} \mathrm{C}$ using a conventional thermocycler PCR (polymerase chain reaction) unit (TECHNE PCR, TC-3000). Immobilization was carried out by casting aptamers solution onto the screen-printed gold electrode surface; the samples were then incubated for $4 \mathrm{~h}$ at room temperature in a $100 \%$ humidity chamber. The unreacted aptamers were removed from the electrode surface by several rinses with non-folding buffer (HBB), then the screen-printed gold electrode with immobilized aptamers were kept in HBB in the fridge to prevent aptamers from coiling. The electrodes with immobilized aptamers could be kept in such conditions for a long time (few weeks) without losing their functionality. The aptamers, which may change their stable secondary structure during storage, can be revived by thermocycling the electrodes immersed in PBB [25]. The electrodes with immobilized aptamers can be also recovered after the use by thermocycling (which causes aptamers to uncoil and release the analyte molecules) and subsequent washing out of the released analytes [25].

\subsection{Electrochemical Measurements}

The measurements of cyclic voltammograms (CVs) were carried out using a DropSTAT4000P potentiostat from DropSens controlled by Autolab software and DropSens screen-printed gold electrodes (SPGEs). These electrodes have a conventional three-electrode configuration with gold working and counter electrodes, and $\mathrm{Ag} / \mathrm{AgCl}$ pseudo-reference electrode. $\mathrm{CV}$ measurements were carried out on electrodes with immobilized aptamers, first, in pure buffer solution (HBB), then in HBB with the sequential addition of different concentrations dopamine, e.g., 0.1, 0.5, 1.0, and $10 \mathrm{nM} \mathrm{L}^{-1}$. Typical incubation time was $3 \mathrm{~min}$, which secured the saturation of the response (see the kinetics study in the experimental results section below). The selected range of concentrations corresponds to typical amounts of dopamine in blood [1,2].

The voltage range between -0.5 and $+0.5 \mathrm{~V}$ was selected for these measurements in order to observe the characteristic peaks of the ferrocene group. The selection of a fixed voltage of $\pm 0.2 \mathrm{~V}$ for 
further analysis allowed monitoring of both the oxidation and reduction currents peaks considering their $0.2 \mathrm{~V}$ expansion on both sides. The aptamer/dopamine binding kinetics at different concentrations of dopamine were studied by recording the time dependence of the cathodic currents on the SPGEs at a fixed potential of $-0.2 \mathrm{~V}$. Control measurements were taken on electrodes without immobilized aptamers. Also, negative control measurements were carried out on aptamer-functionalized electrodes using another analyte molecule, i.e., vitamin C, which is not complementary to the aptamer used.

Electrochemical impedance spectra (EIS) were recorded using an impedance spectroscopy instrument PARSTAT 4000 A and gold interdigitated electrodes (from Metrohm, DropSens) containing 250 fringes on each side spaced by $5 \mu \mathrm{m}$; the overlapping length was $6.76 \mathrm{~mm}$. The AC voltage amplitude was $5 \mathrm{mV}$, and the frequency was varied from $0.1 \mathrm{~Hz}$ to $100 \mathrm{kHz}$; no DC bias was applied. Similar to CV measurements, the EIS measurements were carried out on electrodes both coated and non-coated with aptamers, in buffer solutions containing different concentrations of dopamine and vitamin C (negative control).

Complementary optical characterization of aptamers layers immobilized on 25-nm thick gold films thermally evaporated onto microscopic glass slides was carried out with spectroscopic ellipsometry using a J. A. Woollam M2000VI instrument operating in total internal reflection mode (TIRE) [26]. The process of dopamine binding was monitored by changes in the thickness of the aptamer layer as a result of coiling of the aptamer around the target analyte.

\section{Results}

\subsection{Cyclic Voltammograms (CV)}

Typical CVs recorded on DropSens screen-printed three-electrode assemblies with immobilized aptamer in buffer solutions containing different concentrations of dopamine are shown in Figure 1a. Both anodic and cathodic peaks observed at about 0.4 and $-0.2 \mathrm{~V}$, respectively, are associated with redox activity of ferrocene-labelled aptamers. The relative changes in the amplitudes $\Delta \mathrm{I} / \mathrm{I}=\left(I-I_{\text {ref }}\right) / I_{\text {Iref }}$ of both anodic and cathodic currents are correlated with the concentration of dopamine in buffer as illustrated on the inset in Figure 1a; the increase of dopamine concentration from 0 (pure buffer) to $10 \mathrm{nM} \mathrm{L}^{-1}$ causes increases in both cathodic and anodic currents. The observed saturation of current is caused by the saturation of molecular receptors, e.g., aptamers. Negative control measurements in PBB containing $10 \mathrm{nM}$ of vitamin $\mathrm{C}$, which is not complementary to the aptamer used, showed no response.



(a)

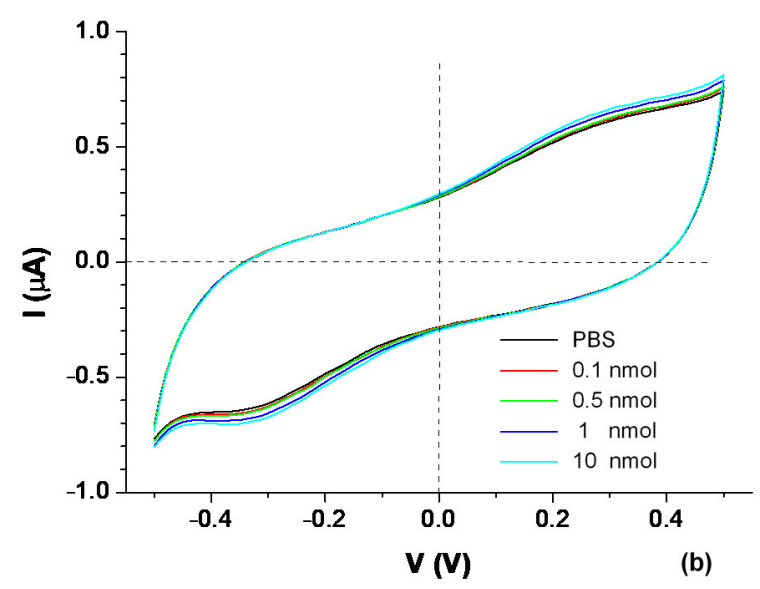

(b)

Figure 1. Typical cyclic voltammograms (CVs) recorded on electrodes with (a) and without (b) immobilized aptamers in PBB containing different concentrations of dopamine. Inset in Figure 2a shows the relative changes of both anodic and cathodic currents vs. dopamine concentration. 




Figure 2. The scheme of electrochemical aptasensing of dopamine.

Control measurements carried out on bare electrodes without immobilized aptamers show very small (less than $1 \mu \mathrm{A}$ ) current, which increases slightly with the increase in dopamine concentration (see Figure 1b) because of the redox activity of dopamine [27]. All measurements were repeated from 3 to 5 times, and typical results are presented.

The results shown in Figure 1a could be understood in terms of the following model. Binding of dopamine causes a change in the aptamer conformation, which brings the ferrocene label closer to the electrode surface, thus enhancing the charge transfer. Such processes illustrated schematically in Figure 2 cause the increase of the amplitudes of characteristic anodic and cathodic current peaks in CVs.

\subsection{Electrochemical Impedance Spectroscopy Measurements (EIS)}

Figure 3a displays typical EIS recorded on interdigitated electrodes with immobilized aptamers immersed in PBB containing different concentrations of dopamine. The Nyquist plots in Figure 3a are almost perfect semi-circles (a typical feature of electrochemical processes not limited by diffusion) with the diameter strongly dependent of dopamine concentration. The increase in dopamine concentration causes both the reduction in Nyquist semi-circle diameter and their shift to lower values of $Z_{R e}$. Such behavior could be explained in terms of a simplified (without diffusion-associated impedance) equivalent circuit model [28] shown as an inset in Figure 3a.

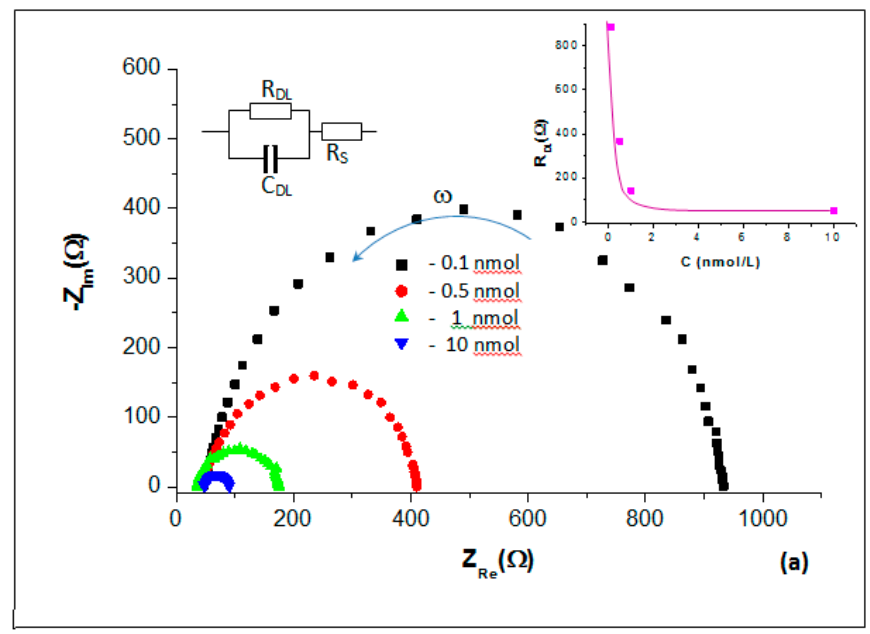

(a)

Figure 3. Cont. 


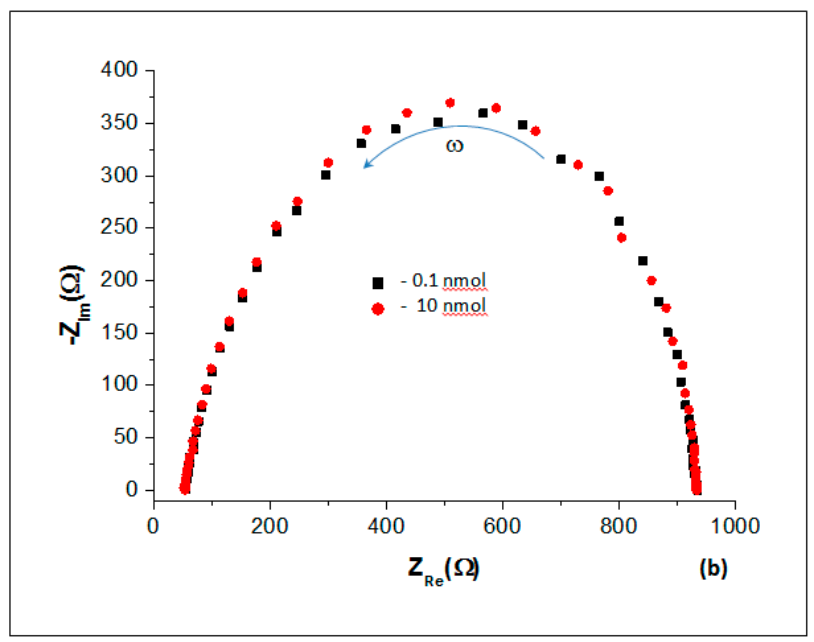

(b)

Figure 3. Nyquist plots of electrochemical impedance spectra (EIS) data on electrodes with (a) and without (b) immobilized aptamers at different concentrations of dopamine. Arrows show the direction of frequency increase. Insets in Figure 3a shows the equivalent circuit model (left) and the dependence of $R_{D L}$ on dopamine concentration (right).

According to this model, $Z \_\operatorname{Re}^{\wedge} 0=\mathrm{R} \_\mathrm{DL}+\mathrm{R} \_\mathrm{S}$ at low frequencies $(\omega=0$, far right end of Nyquist plot), while $Z \_R e^{\wedge} \infty=R \_S$ at high frequencies $(\omega=\infty$, far left end of Nyquist plot) [28], where RDL and RS are respectively the resistances of a double layer and electrolyte solution. Therefore, the characteristic parameter of RDL can be found by subtracting $Z$ values at lowest and highest frequencies: $\mathrm{R} \_D L=Z \_\operatorname{Re}^{\wedge} 0-Z \_\operatorname{Re}^{\wedge} \infty$. The dependence of RDL on the concentration of dopamine is shown as inset in Figure 3a. The reduction in RDL upon increase in dopamine concentration is in line with the CV results as well as with the electrochemical aptasensing model in Figure 2.

EIS measurements on electrodes without immobilized aptamers in buffer solution with the lowest $\left(0.1 \mathrm{nM} \mathrm{L}^{-1}\right)$ and highest $\left(10 \mathrm{nM} \mathrm{L}^{-1}\right)$ concentrations of dopamine show practically no changes in Nyquist plots (see Figure 3b), which confirms the CVs results in Figure 1b. Negative control experiments using a non-complementary to the aptamer used target molecule (vitamin C) showed no changes in the EIS graphs. The EIS measurements were repeated from 3 to 5 times, and typical results were presented.

\subsection{Evaluation of Low Detection Limit}

The values of low detection limit (LDL) in CV and EIS measurements were estimated, respectively, from the dependencies of relative changes of current $(\Delta I / I)$ and double layer conductance $\left(1 / R_{D L}\right)$ against the total concentration of dopamine in semi-logarithmic coordinates shown in Figure 4. Because both CV and EIS measurements were carried out in a series of scans with sequential increasing of dopamine concentrations (i.e., $0.1,0.5,1$, and $10 \mathrm{nM}$ ), the total concentrations of dopamine bound to aptamer were $0.1,0.6,1.6$, and $11.6 \mathrm{nM}$.

The accuracy of $\mathrm{CV}$ measurements was in the range of $1 \%$, thus LDL was found by linear extrapolation of the $\Delta I / I$ vs. $C$ dependence (Figure $4 a$ ) to a triple level of noise estimated as 0.03 . The LDL values of $60 \mathrm{pM}$ and $90 \mathrm{pM}$ were found for anodic and cathodic current, respectively. The EIS measurements allowed the evaluation of $R_{D L}$ with the accuracy of about $1 \Omega$. For LDL evaluation, it is more convenient to use the reciprocal value of a double layer conductance $\left(S_{D L}=1 / R_{D L}\right)$. Using the values of $R_{D L}$ about $1 \mathrm{k} \Omega$ at low concentrations of dopamine, the $S_{D L}$ noise level can be estimated as $\Delta S_{D L}=\Delta R_{D L} / R_{D L}^{2}=10^{-5} S$ or $0.01 \mathrm{mS}$, which is practically a zero level in Figure $4 \mathrm{~b}$. The intercept of the linear approximation of $1 / R_{D L}$ vs. $C$ graph at low concentrations yields the LDL value of $20 \mathrm{pM}$ for EIS measurements. The above LDL estimations showed that the EIS method is more sensitive as compared to CVs. 

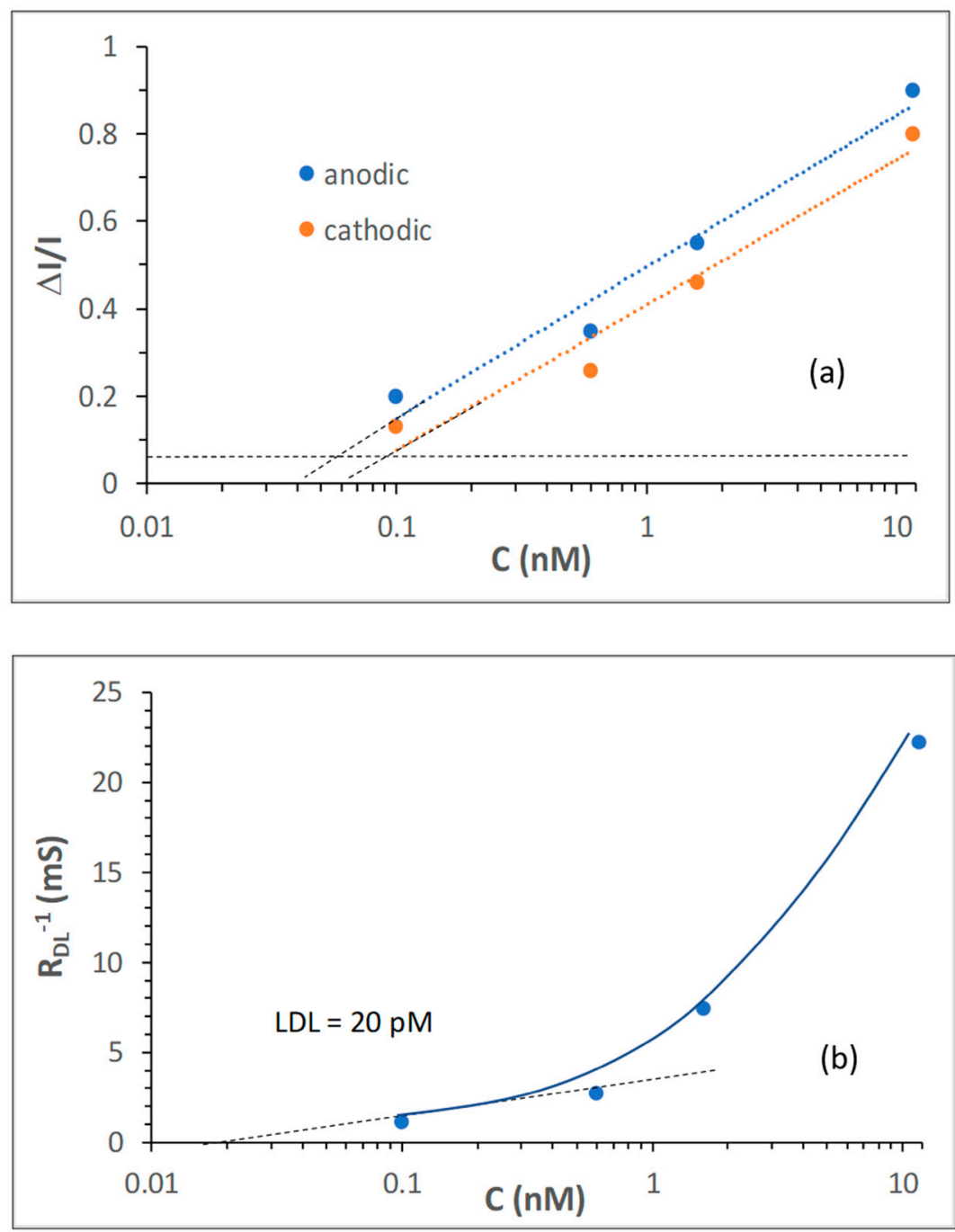

Figure 4. Evaluation of low detection limit (LDL) in CV (a) and EIS (b) measurements.

\subsection{The Study of Aptamer-Dopamine Binding Kinetics}

Typical time dependencies of cathodic current (at $-0.2 \mathrm{~V}$ ) at different concentrations of dopamine are shown in Figure 5. As one can see, the increase in dopamine concentration leads to faster saturation and smaller current changes. The full saturation of responses was practically achieved in $60 \mathrm{~s}$, which justified the $3 \mathrm{~min}$ incubation time in CV and EIS measurements. The analysis of binding kinetics was carried out using the differential equation for adsorption/desorption processes on molecular binding sites on the surface $[29,30]$, which is particularly suitable for a monolayer of aptamers immobilized on the surface of screen-printed gold electrodes. According to this approach, the binding kinetics is best described by a rising exponential function with the reciprocal time constant $(1 / \tau)$ dependent of analyte concentration $(C)$ as:

$$
I=I_{o}\left(1-e^{-\frac{t}{\tau}}\right) ; \frac{1}{\tau}=k_{a} C+k_{d}
$$

where $k_{a}$ and $k_{d}$ are, respectively, the rates of adsorption and desorption. The values of time constant $(\tau)$ were found at every concentration of dopamine, and the linear plot of $1 / \tau$ vs. C is shown as inset in Figure 5. The values of $k_{a}$ and $k_{d}$ were obtained from that graph as the gradient and intercept, respectively. Then both the association $\left(K_{A}\right)$ and affinity $\left(K_{D}\right)$ constants of binding can be determined as:

$$
K_{A}=k_{a} / k_{d} \text { and } K_{D}=1 / K_{A}
$$




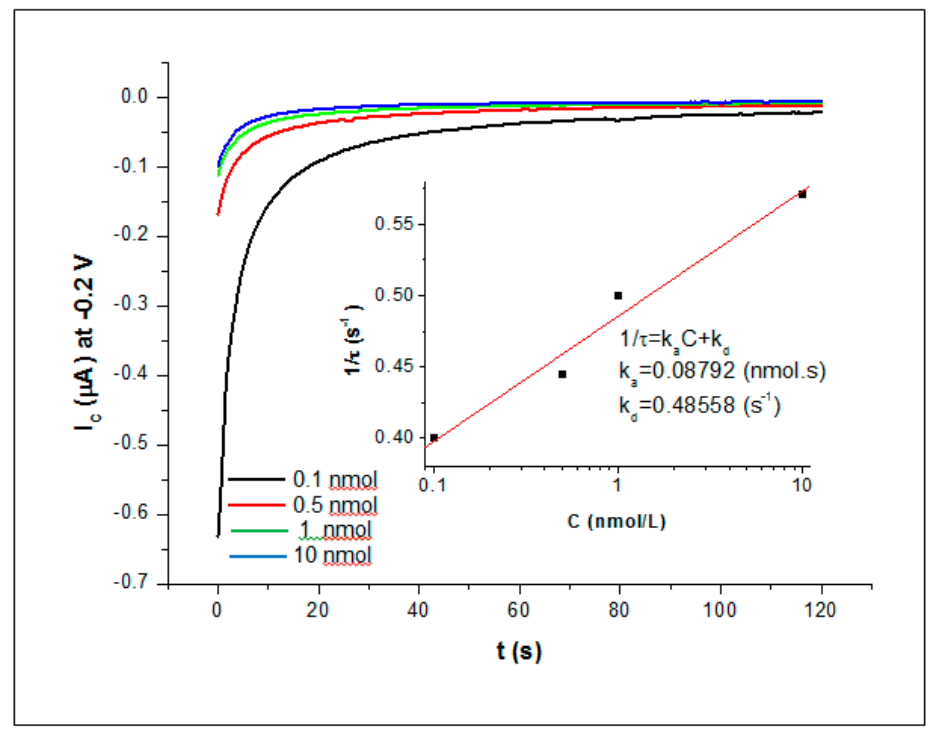

Figure 5. Time dependencies of Ic (at $-0.2 \mathrm{~V}$ ) at different concentrations of dopamine. Inset shows the linear dependence of $1 / \tau$ vs., $C$ and the values of $k_{a}$ and $k_{d}$ found.

The obtained values for aptamers-dopamine binding are $K_{A}=1.81 \times 10^{8} \mathrm{M}^{-1}$ and $K_{D}=5.52 \times 10^{-9} \mathrm{M}$ or $5.52 \mathrm{nM}$, which confirmed the high affinity of the aptamer used, similar to that of antibodies.

\subsection{Spectroscopic Ellipsometry Measurements}

The method of total internal reflection ellipsometry (TIRE) was used in this work as a complementary technique to confirm the process of binding dopamine to aptamer. The method of TIRE being a combination of SPR (surface plasmon resonance) and spectroscopic ellipsometry proved to be particularly useful for detection of small molecules, such as mycotoxins, in direct assays either with specific antibodies or aptamers, as was outlined earlier in several publications, for example [26]. The schematic diagram of TIRE is shown as inset in Figure 6. In contrast to traditional SPR which records the amplitude of reflected p-polarized light, in TIRE, both the amplitude-related parameter $\Psi$ and phase-related parameter $\Delta$ are recorded with the latter being much more sensitive to changes in the film thickness [26]. A series of TIRE measurements were carried out on samples of thin $(25 \mathrm{~nm})$ gold films evaporated onto glass slides with dopamine-specific aptamers immobilized on the gold surface, a typical set of $\Delta$ spectra are shown in Figure 6.

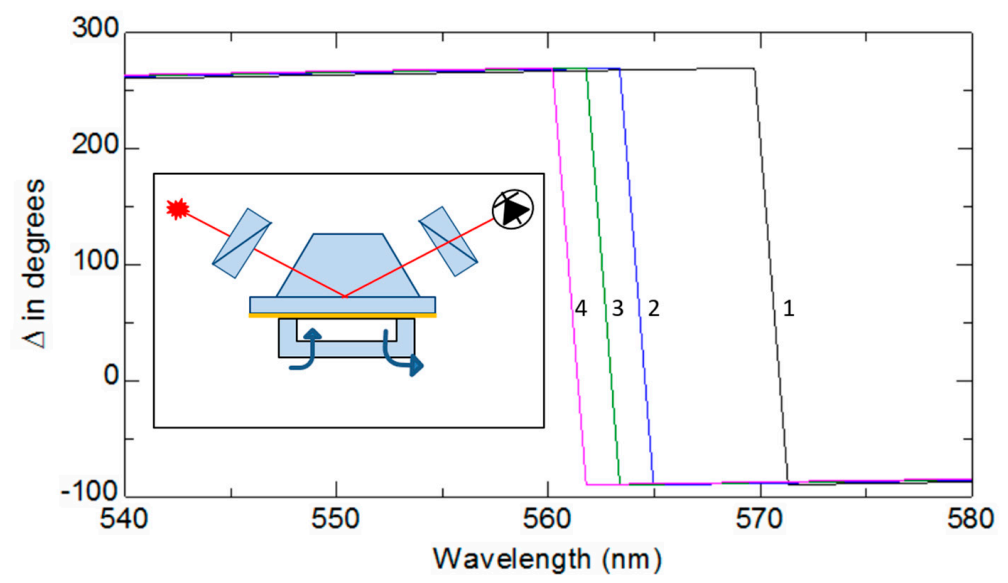

Figure 6. Typical series of total internal reflection ellipsometry (TIRE) $\Delta$-spectra recorded in phosphate binding buffer (PBB) on aptamer layer (1) and after consecutive steps of binding dopamine at $0.1 \mathrm{nM}$ (2), $1 \mathrm{nM}(3)$, and $10 \mathrm{nM}$ (4) concentrations. 
As can be seen, the spectra of $\Delta$ progressively shift to the left (to shorter wavelengths) after binding dopamine, which corresponds to decrease in the aptamer layer thickness. These results confirmed once again the model of electrochemical aptasensing outlined earlier and presented in Figure 2.

\section{Conclusions}

The research carried out proved a concept of electrochemical detection of dopamine using a specific aptamer labeled with ferrocene and immobilized on the surface of gold screen-printed electrodes via a thiol group. Both the CV and EIS measurements were capable of detecting dopamine in low concentrations in the range from $10 \mathrm{nM}$ to $0.1 \mathrm{nM}$. The LDL (low detection limit) estimated at the relative current level corresponding to the triple level of noise is about $60 \mathrm{pM}$ for anodic current and $90 \mathrm{pM}$ for cathodic current measurements; LDL for EIS measurements was estimated as $20 \mathrm{pM}$. The estimated LDL values are lower than those reported earlier in [18] and [19]. It also shows that the EIS method is superior to CVs in terms of sensitivity, and perhaps, more promising for future sensor development.

The study of aptamer-dopamine binding kinetics revealed a very high specificity of binding with an affinity constant $K_{D}$ of about $5 \mathrm{nM}$. The obtained values of $K_{D}$ indicate high selectivity of the aptamers used, therefore a zero response to a non-complementary target such as vitamin $C$ is not surprising. Independent optical measurements using TIRE confirmed the reduction in the aptamer layer thickness caused by changes in their secondary structure caused by dopamine binding.

A simple and cost-effective electrochemical aptasensing technology outlined in this work could be very promising for the detection of dopamine in human body fluids as a possible diagnostic tool for the early detection of Parkinson's disease as well as other neurological disorders such as schizophrenia and Alzheimer's disease. Further thorough investigation is required to fulfil such ambitious tasks, which should include stability, reproducibility, and additional cross-sensitivity tests, and, of course, the detection of dopamine in a complex media including real samples of blood serum or other body fluids.

Author Contributions: H.A.-A. and C.O. carried out majority of experimental work, F.D. and N.W. developed the concept and involved in discussion and writing, A.N. was involved in all aspects of work, but mostly in data analysis and writing. All authors have read and agreed to the published version of the manuscript.

Funding: There was no funding dedicated to this work.

Acknowledgments: Cansu Ozkaya would like to thank the ERASMUS program for supporting her research visit to MERI, SHU. Hisham Abu-Ali expresses gratitude to the Ministry of High Education of Iraq for sponsoring his PhD study at Sheffield Hallam University.

Conflicts of Interest: There is no conflict of interests.

\section{References}

1. Conrad, Brian, The Role of Dopamine as a Neurotransmitter in the Human Brain, Neuroscience. Available online: http://www.enzolifesciences.com/science-center/technotes/2018/november/the-role-of-dopamineas-a-neurotransmitter-in-the-human-brain/ (accessed on 9 November 2018).

2. Tavakolian-Ardakani, Z.; Hosu, O.; Cristea, C.; Mazloum-Ardakani, M.; Marrazza, G.; Tavakolian-Ardakani, Z.; Hosu, O.; Mazloum-Ardakani, M. Latest Trends in Electrochemical Sensors for Neurotransmitters: A Review. Sensors 2019, 19, 2037. [CrossRef]

3. Tukimin, N.; Abdullah, J.; Sulaiman, Y. Review-Electrochemical Detection of Uric Acid, Dopamine and Ascorbic. Acid. J. Electrochem. Soc. 2018, 165, B258-B267. [CrossRef]

4. Ku, S.; Palanisamy, S.; Chen, S.-M. Highly selective dopamine electrochemical sensor based on electrochemically pretreated graphite and nafion composite modified screen printed carbon electrode. J. Colloid Interface Sci. 2013, 411, 182-186. [CrossRef] [PubMed]

5. Zhao, J.; Yu, Y.; Weng, B.; Zhang, W.; Harris, A.T.; Minett, A.; Yue, Z.; Huang, X.-F.; Chen, J. Sensitive and selective dopamine determination in human serum with inkjet printed Nafion/MWCNT chips. Electrochem. Commun. 2013, 37, 32-35. [CrossRef] 
6. Walch, N.J.; Davis, F.; Langford, N.; Holmes, J.L.; Collyer, S.D.; Higson, S.P.J. Enhancement of Electrode Performance by a Simple Casting Method Using Sonochemically Exfoliated Graphene. Anal. Chem. 2015, 87, 9273-9279. [CrossRef]

7. Ai, S.; Chen, Y.; Liu, Y.; Zhang, Q.; Xiong, L.; Huang, H.; Li, L.; Yu, X.; Wei, L. Facile synthesis of nitrogen-doped graphene aerogels for electrochemical detection of dopamine. Solid State Sci. 2018, 86, 6-11. [CrossRef]

8. Yang, Y.J.; Li, W. CTAB functionalized graphene oxide/multiwalled carbon nanotube composite modified electrode for the simultaneous determination of ascorbic acid, dopamine, uric acid and nitrite. Biosens. Bioelectron. 2014, 56, 300-306. [CrossRef]

9. Palanisamy, S.; Ku, S.; Chen, S.-M. Dopamine sensor based on a glassy carbon electrode modified with a reduced graphene oxide and palladium nanoparticles composite. Microchim. Acta 2013, 180, 1037-1042. [CrossRef]

10. Niu, L.M.; Lian, K.Q.; Shi, H.-M.; Wu, Y.B.; Kang, W.; Bi, S.Y. Characterization of an ultrasensitive biosensor based on a nano-Au/DNA/nano-Au/poly(SFR) composite and its application in the simultaneous determination of dopamine, uric acid, guanine, and adenine. Sens. Actuators B: Chem. 2013, 178, 10-18. [CrossRef]

11. Fabregat, G.; Armelin, E.; Alemán, C. Selective Detection of Dopamine Combining Multilayers of Conducting Polymers with Gold Nanoparticles. J. Phys. Chem. B 2014, 118, 4669-4682. [CrossRef]

12. Tsierkezos, N.G.; Ritter, U.; Thaha, Y.N.; Knauer, A.; Fernandes, D.; Kelarakis, A.; McCarthy, E.K. Boron-doped multi-walled carbon nanotubes as sensing material for analysis of dopamine and epinephrine in presence of uric acid. Chem. Phys. Lett. 2018, 710, 157-167. [CrossRef]

13. Yan, X.; Gu, Y.; Li, C.; Zheng, B.; Li, Y.; Zhang, T.; Zhang, Z.; Yang, M. Morphology-controlled synthesis of $\mathrm{Bi}_{2} \mathrm{~S}_{3}$ nanorods-reduced graphene oxide composites with high-performance for electrochemical detection of dopamine. Sens. Actuators B Chem. 2018, 257, 936-943. [CrossRef]

14. Kim, D.-S.; Kang, E.-S.; Baek, S.; Choo, S.-S.; Chung, Y.-H.; Lee, D.; Min, J.; Kim, T.-H. Electrochemical detection of dopamine using periodic cylindrical gold nanoelectrode arrays. Sci. Rep. 2018, 8, 14049. [CrossRef] [PubMed]

15. Jarczewska, M.; Gorski, L.; Malinowska, E. Electrochemical aptamer-based biosensors as potential tools for clinical diagnostics. Anal. Methods 2016, 8, 3861-3877. [CrossRef]

16. Dunn, M.; Jimenez, R.M.; Chaput, J.C. Analysis of aptamer discovery and technology. Nat. Rev. Chem. 2017, 1, 76. [CrossRef]

17. Zhang, Y.; Lai, B.S.; Juhas, M. Recent Advances in Aptamer Discovery and Applications. Molecules 2019, 24, 941. [CrossRef] [PubMed]

18. Zheng, Y.; Wang, Y.; Yang, X. Aptamer-based colorimetric biosensing of dopamine using unmodified gold nanoparticles. Sens. Actuators B Chem. 2011, 156, 95-99. [CrossRef]

19. Xu, J.; Li, Y.; Wang, L.; Huang, Y.; Liu, D.; Sun, R.; Luo, J.; Sun, C. A facile aptamer-based sensing strategy for dopamine through the fluorescence resonance energy transfer between rhodamine B and gold nanoparticles. Dye. Pigment. 2015, 123, 55-63. [CrossRef]

20. Nakatsuka, N.; Cao, H.H.; Deshayes, S.; Melkonian, A.L.; Kasko, A.M.; Weiss, P.S. Andrews Aptamer Recognition of Multiplexed Small-Molecule-Functionalized Substrates. Appl. Mater Interfaces 2018, 10, 23490-23500. [CrossRef]

21. Farjami, E.; Campos, R.; Nielsen, J.S.; Gothelf, K.V.; Kjems, J.; Ferapontova, E.E. RNA Aptamer-Based Electrochemical Biosensor for Selective and Label-Free Analysis of Dopamine. Anal. Chem. 2013, 85, 121-128. [CrossRef]

22. Xu, Y.; Hun, X.; Liu, F.; Wen, X.; Luo, X. Aptamer biosensor for dopamine based on a gold electrode modified with carbon nanoparticles and thionine labeled gold nanoparticles as probe. Microchim. Acta 2015, 182, 1797-1802. [CrossRef]

23. Azadbakht, A.; Roushani, M.; Abbasi, A.R.; Derikvand, Z. Design and characterization of electrochemical dopamine aptamer as convenient and integrated sensing platform. Anal. Biochem. 2016, 507, 47-57. [CrossRef] [PubMed]

24. Rhouati, A.; Yang, C.; Hayat, A.; Marty, J.-L. Aptamers: A promising tool for ochratoxin A detection in food analysis. Toxins 2013, 5, 1988-2008. [CrossRef] [PubMed] 
25. Abu-Ali, H.; Nabok, A.; Smith, T.J. Development of novel and highly specific ssDNA-Aptamer-based electrochemical biosensor for rapid detection of Mercury (II) and Lead (II) ions in water. Chemosensors 2019, 7, 27. [CrossRef]

26. Nabok, A.; Tsargorodskaya, A. The method of total internal reflection ellipsometry for thin film characterization and sensing. Thin Solid Films 2008, 516, 8993-9001. [CrossRef]

27. Chen, S.-M.; Peng, K.-T. The electrochemical properties of dopamine, epinephrine, norepinephrine, and their electrocatalytic reactions on cobalt(II) hexacyanoferrate films. J. Electroanal. Chem. 2003, 547, 179-189. [CrossRef]

28. Bonanos, N.; Steele BC, H.; Butler, E.P.; Macdonald, J.R.; Johnson, W.B.; Worrell, W.L.; McKubre, M.C. Applications of impedance spectroscopy. Impedance spectroscopy: Theory, experiment, and applications. History 2018, 1, 1-13.

29. Nabok, A.; Tsargorodskaya, A.; Mustafa, M.K.; Szekacs, I.; Starodub, N.F.; Szekacs, A. Detection of low molecular weight toxins using optical phase detection techniques. Sens. Actuators B Chem. 2011, 154, $232-237$. [CrossRef]

30. Battaglioli, G.; Liu, H.; Martin, D.L. Kinetic differences between the isoforms of glutamate decarboxylase: Implications for the regulation of GABA synthesis. J. Neurochem. 2003, 86, 879-887. [CrossRef]

(C) 2020 by the authors. Licensee MDPI, Basel, Switzerland. This article is an open access article distributed under the terms and conditions of the Creative Commons Attribution (CC BY) license (http://creativecommons.org/licenses/by/4.0/). 